\title{
Nationwide seroprevalence of antibodies against SARS-CoV-2 in Israel
}

\author{
Shay Reicher ${ }^{1}\left(\mathbb{D} \cdot\right.$ Ronit Ratzon $^{1} \cdot$ Shay Ben-Sahar ${ }^{2,3} \cdot$ Sharon Hermoni-Alon $^{4} \cdot$ David Mossinson $^{5} \cdot$ Yotam Shenhar $^{6}$. \\ Michael Friger $^{7} \cdot$ Yaniv Lustig $^{8} \cdot$ Sharon Alroy-Preis $^{1} \cdot$ Emilia Anis $^{1} \cdot$ Siegal Sadetzki $^{3,9} \cdot$ Ehud Kaliner $^{1}$
}

Received: 31 December 2020 / Accepted: 8 April 2021 / Published online: 21 April 2021

(c) Springer Nature B.V. 2021

\begin{abstract}
The first local spread of COVID-19 in Israel was detected in March 2020. Due to the diversity in clinical presentations of COVID-19, diagnosis by RT-PCR alone might miss patients with mild or no symptoms. Serology testing may better evaluate the actual magnitude of the spread of infection in the population. This is the first nationwide seroprevalence study conducted in Israel. It is one of the most widespread to be conducted thus far, and the largest per-country population size. The survey was conducted between June 28 and September 14, 2020 and included 54,357 patients who arrived at the Health Maintenance Organizations to undergo a blood test for any reason. A patient was considered seropositive after two consecutive positive results with two different kits (Abbott and DiaSorin). The overall seroprevalence was 3.8\% (95\% CI 3.7-4.0), males higher than females [4.9\% (95\% CI 4.6-5.2) vs. 3.1\% (95\% CI 2.9-3.3) respectively]. Adolescents had the highest prevalence [7.8\% (95\% CI 7.0-8.6)] compared to other age groups. Participants who had undergone RT-PCR testing had a tenfold higher risk to be seropositive. The prevalence-to-incidence ratio was 4.5-15.7. Serology testing is an important complimentary tool for assessing the actual magnitude of infection and thus essential for implementing policy measures to control the pandemic. A positive serology test result was recently accepted in Israel as being sufficient to define recovery, with possible far-reaching consequences, such as the deploying of employees to ensure the maintenance of a functional economy.
\end{abstract}

Keywords COVID-19 $\cdot$ Serology $\cdot$ Seroprevalence $\cdot$ SARS-CoV-2 $\cdot$ Epidemiology

\section{Introduction}

COVID-19 is caused by the novel SARS-CoV-2 virus [1]. SARS-CoV-2 infection can be clinically manifested in varying degrees of severity, from asymptomatic infection to serious illness, and sometimes death. The first local case of

Shay Reicher and Ronit Ratzon are the first authors equally contributed to this work.

Siegal Sadetzki, Ehud Kaliner are the senior authors equally contributed to this work.

Shay Reicher

shay.reicher@moh.gov.il

1 Public Health Services, Ministry of Health, 39 Yirmiyahu Street, Jerusalem, Israel

2 Schneider Children's Medical Center, Clalit Research Institute, Petach Tikva, Israel

3 Sackler Faculty of Medicine, Tel Aviv University, Tel Aviv, Israel

4 Maccabi Healthcare Group, 27 Hamered, Tel Aviv, Israel
COVID-19 in Israel was detected in March 2020. Since then, Israel experienced two major waves of COVID-19 outbreaks. A total of 413,004 individuals underwent RT-PCR testing from February 21, 2020 until the end of May 2020, from which 17,078 cases were confirmed (a $4.1 \%$ positive rate). In May 2020, the influence of the commercial, social, and travel restrictions brought the number of detected cases to less than 15 per day. The second wave started in June 2020. By September 2020, a peak of over 8000 newly confirmed cases were detected daily. From June 1 to September 14, 2020, 1,626,064 individuals underwent RT-PCR testing, and

5 Meuhedet Health Services, 124 Ibn Gvirol Street, Tel Aviv, Israel

6 Leumit Health Services, 3 Ariel Sharon Street, Or Yehuda, Israel

7 Faculty of Health Sciences, Ben-Gurion University of the Negev, Be'er Sheva, Israel

8 Central Virology Laboratory, Ministry of Health and Sheba Medical Center, Tel-Hashomer, Israel

9 Gertner Institute, Tel-Hashomer, Israel 
159,435 new cases were confirmed (a $9.8 \%$ positive rate) [2]. Up to September 14, 2020 6,983,391 were infected worldwide with a reported death rate of $4.14 \%$ [3].

Under the National Health Insurance law [4] all Israeli citizens must be registered in one of the country's four Health Maintenance Organizations (HMOs). HMO community healthcare services are widespread throughout the country.

Serologic testing may serve as an internationally important tool for evaluating the genuine rate of all (those who were diagnosed and those who were not) infected individuals in the community regardless of their clinical presentation of the disease (symptomatic or asymptomatic). Individuals with or without symptoms who have not been tested by RTPCR due to lack of awareness or willingness to be tested, as well as due to limited access to medical services or limited availability of tests, may lead to an underestimation of the extent of the COVID-19 burden in a country. Therefore, serology testing may better evaluate the actual magnitude of infection.

Worldwide, several sero-epidemiological studies were conducted [5-9] utilizing serological tests to reveal the true extent of COVID-19 infection in the general population. The sero-epidemiological surveys conducted thus far included a sample to population ratio of: $0.19 \%$ in England, [7] 0.1\% in Spain, [5] 0.05\% in US [9] and Sweden [8] and 0.026\% in Brazil [6]. Compared to other studies conducted thus far, the current study covers much greater proportions of the target population, $0.58 \%$ sample to population ratio. This is the first nationwide seroprevalence study conducted in Israel. The vast and well-established data obtained in the current study might contribute to determination of testing strategies and policy decision making in other countries as well.

\section{Methods}

\section{Study design and participants}

This study was approved by the legal department of the Israeli Ministry of Health. The Israeli first nationwide seroepidemiologic survey began on June 28, 2020 and ended on September 14, 2020. In order to obtain an appropriate representation of the entire population, all municipalities in Israel were characterized according to the following parameters: ethnicity, religious affiliation, socioeconomic status, number of residents, and geographical location (district). The study population included insured individuals who arrived at the HMOs to undergo a blood test for any reason. Due to technical difficulties, the HMOs were only able to provide the number of performed tests and not the number of referred participants. The final sample included all participants that underwent the serology testing, excluding participants with missing data (Fig. 1). For further information, see Online Resource methods.

A 3-5 ml blood sample was collected in a standard vacuum gel tube. Data on the participant's sex, age, and home address were retrieved from the medical file. Data on RT-PCR status, including date performed and result, were obtained from the Ministry of Health Registry.

\section{Detection of SARS-CoV-2 antibodies}

Two serologic tests were used, both using qualitative detection of IgG antibodies to SARS-CoV-2: SARS-CoV-2 IgG assay by Abbott ${ }^{\circledR}$ and LIAISON® SARS-CoV-2 S1/S2 IgG by DiaSorin. Specificity and sensitivity of the analytical methods were calculated at the Israeli Central Virology Lab and described in details elsewhere [10].

In order to achieve the highest specificity it was determined that the sample would be analyzed with the Abbott assay and, if found to be positive, would be validated with the DiaSorin assay. Only participants with a positive result in both tests were classified as being seropositive for SARSCov-2. The combined specificity after utilizing both tests was $99.99 \%$ (95\% CI 99.8-100.0). In accordance, due to the double assay test, the combined sensitivity was reduced to 76.7\% (95\%CI 73.9-80.0).

\section{Statistical analysis}

We estimated seroprevalence as being the proportion of individuals who had positive serology results within the survey's population and assessed the effect of selected risk factors and determinants (age, sex, RT-PCR status, time period, and municipality characteristics) on the probability to receive a seropositive result. The serology test result was compared to the RT-PCR result in order to determine the RT-PCR capability as a predictor for receiving a seropositive result. Due to the changes in the epidemiological curve during the survey period, the analysis was divided into two-week periods when relevant. Since all of the municipalities included in

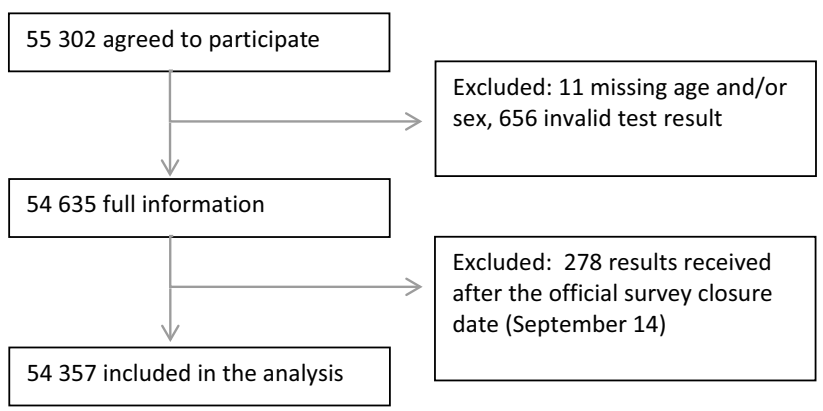

Fig. 1 Flowchart of participants in the seroepidemiological nationwide survey for SARS-CoV-2 
the study were divided into strata according to their characteristics, we approached each municipal characteristic as an independent parameter in the univariate analysis.

The chi square was applied for the univariate analysis, when appropriate, and 95\% CIs were calculated with the Clopper-Pearson interval. The seroprevalence-associated risk factors were examined with a multivariate generalized linear mixed model (MGLMM). The MGLMM was fitted with age, sex, time period, and RT-PCR status as fixed effects, and the municipal strata were taken as a random effect. The variance component was selected as the random effect covariance. Receiver operating characteristic (ROC) curves were created from which area under the ROC curve (AUC) was calculated as a metric of model performance.

In order to reflect the seroprevalence of the general population of Israel, we used sampling weights for sex, age, municipal strata and RT-PCR status. For the weights procedure, we weighted the age by sex distribution for each municipal stratum to that of the general population. Data regarding the general population was obtained from the Israeli Central Bureau of Statistics. The statistical analysis was performed with the IBM SPSS Statistics for Windows, Version 20.0. Armonk, NY: IBM Corp.

\section{Results}

During the recruitment period, 55,302 individuals consented to participate in the survey, and 54,357 of them were included in the final analysis (Fig. 1). The study population consisted of $58.6 \%$ males. The mean age of the entire cohort was 45.6 years ( \pm standard deviation 19.83) (Online Resource Table 1). The overall survey seroprevalence was $3.8 \%$ (95\% CI 3.7-4.0). After weighing for age, sex, and RTPCR status, the overall seroprevalence was $4.6 \%$ (95\% CI 4.4-4.8).

Table 1 demonstrates the seroprevalence according to sociodemographic characteristics. Males had a higher prevalence compared to females ( $4.9 \%$ vs. $3.1 \%, p<0.001)$. The highest seroprevalence was among children (5.6\%) and adolescents (7.8\%). The highest seroprevalence within the adolescent group was among those between 10 and 13 years of age $(8.6 \%, 95 \% \mathrm{CI} 7.1-10.4)$, with a slightly lower rate among those who were $14-19$ y of age $(7.4 \%, 95 \% \mathrm{CI}$ 6.5-8.4) (Online Resource Table 2).

The seroprevalence decreased as the socioeconomic status increased, from $6.5 \%$ in the low socioeconomic municipalities to $1.6 \%$ in the high socioeconomic municipalities $(p<0.001)$. The prevalence among participants living in large municipalities was higher than that in medium and small municipalities.

The seroprevalence increased over time, along with the rise of the epidemic curve during the study period, from
$2.7 \%$ in the first two weeks to $16 \%$ in the last two weeks (Online Resource Fig. 1). Comparison of the percentages of participants who tested positive both by RT-PCR and serology according to the time interval between the two tests revealed the highest seroprevalence rate between four to eight weeks after a positive RT-PCR test (83.8\%) (Online Resource Table 3). Between 0 and 4 weeks after a positive RT-PCR result, only $63.6 \%$ of the samples had detectible IgG antibodies. Due to the difference in the prevalence according to the time interval between the two tests, it was decided that only RT-PCR tests that had been conducted at least 4 weeks prior to the serology test would be included in further analysis.

A comparison of the seroprevalence according to RTPCR status revealed that the seroprevalence rate among the participants who underwent RT-PCR testing was higher compared to those who did not, regardless of their RT-PCR result ( 21.9 vs. $2.1 \%$, respectively, $p<0.001)$. In total, $74 \%$ of the participants who had a positive RT-PCR result were also positive for the serologic test that had been carried out at least 4 weeks after the RT-PCR test (Table 2).

We then stratified the data of the individuals who underwent both RT-PCR and serology tests into five periods of two weeks. The percentage of participants that underwent RT-PCR testing increased with time, from $7.6 \%$ at the first two weeks of the survey to $28.5 \%$ at the last two weeks. At the respective time intervals, the seroprevalence increased from 18 to $35 \%(p<0.001)$. The percentage of people who had RT-PCR positive test results increased from approximately $18 \%$ at the first two weeks of the survey to approximately $37 \%$ at the last two weeks (Online Resource Table 4).

Table 3 presents the results from a multiple generalized linear mixed model demonstrating the odds ratio (OR) for a positive serology test result. Participants with previous RTPCR results had an 11-fold higher probability of being serologically positive. Males had 1.5 higher odds $(\mathrm{OR}=1.56$, $p<0.001$ ) to be seropositive in comparison to females. For adolescents aged 10-19 years, the odds to be seropositive increased by 3 -fold in comparison to adults 70 years of age or more $(\mathrm{OR}=3.3, p<0.001)$. Participants who were tested during the last two weeks of the current survey had increased odds (Adj OR $=5.4, p<0.001$ ) to be seropositive in comparison to participants that underwent serology testing during the first two weeks of the survey. In a ROC analysis, the AUC was 0.87 ( $p<0.001,95 \%$ CI 0.86-0.88), demonstrating high compatibility of the model.

In order to examine the effect of a given municipality's socioeconomic status as a risk factor for a positive serology test result, we stratified the data by socioeconomic status and used a multivariable logistic analysis. Neither age nor sex differ when a high socioeconomic status was compared to medium and low socioeconomic statuses (Online Resource Table 5). 
Table 1 SARS-CoV-2 seroprevalence by population characteristics

\begin{tabular}{|c|c|c|c|}
\hline Variable & $\begin{array}{l}\text { Number of partici- } \\
\text { pants, n (\%) }\end{array}$ & Seroprevalence \% (95\%CI) & $P$ value \\
\hline Overall & 54,357 & $3.8(3.7-4.0)$ & NA \\
\hline \multicolumn{4}{|l|}{$\operatorname{Sex}$} \\
\hline Male & $22,518(58.6)$ & $4.9(4.6-5.2)$ & \multirow[t]{2}{*}{$<0.001$} \\
\hline Female & $31,839(41.4)$ & $3.1(2.9-3.3)$ & \\
\hline \multicolumn{4}{|l|}{ Age, years by group } \\
\hline $0-9$ & $1699(3.1)$ & $5.6(4.5-6.8)$ & \multirow[t]{8}{*}{$<0.001$} \\
\hline $10-19$ & $4165(7.7)$ & $7.8(7.0-8.6)$ & \\
\hline $20-29$ & $7049(13.0)$ & $4.8(4.3-5.3)$ & \\
\hline $30-39$ & $8556(15.7)$ & $3.7(3.3-4.1)$ & \\
\hline $40-49$ & $8856(16.3)$ & $4.1(3.7-4.6)$ & \\
\hline $50-59$ & $8348(15.4)$ & $3.7(3.3-4.1)$ & \\
\hline $60-69$ & $8750(16.1)$ & $2.6(2.3-2.9)$ & \\
\hline $70+$ & $6937(12.8)$ & $1.7(1.4-2.0)$ & \\
\hline \multicolumn{4}{|c|}{ Municipality ethnicity and religious affiliation ${ }^{a}$} \\
\hline Jewish municipalities & $35,541(71.6)$ & $3.6(3.4-3.8)$ & \\
\hline Ultraorthodox & $2939(7.6)$ & $11.8(10.7-13.0)$ & \multirow[t]{5}{*}{$<0.001$} \\
\hline Non-ultraorthodox & $23,766(61.1)$ & $2.1(1.9-2.3)$ & \\
\hline Mixed & $8836(22.7)$ & $4.7(4.2-5.1)$ & \\
\hline Non-Jewish municipalities & $4454(8.6)$ & $2.1(1.7-2.6)$ & \\
\hline Mixed municipalities & $10,720(19.7)$ & $5.5(5.1-6.0)$ & \\
\hline Non-ultraorthodox & $5919(55.2)$ & $1.8(1.5-2.2)$ & \multirow[t]{2}{*}{$<0.001$} \\
\hline Mixed & $4801(44.8)$ & $10.1(9.2-11)$ & \\
\hline \multicolumn{4}{|c|}{ Socio-economic status of municipalities ${ }^{a}$} \\
\hline High & $11,611(21.4)$ & $1.6(1.4-1.9)$ & \multirow[t]{3}{*}{$<0.001$} \\
\hline Medium & $21,037(38.7)$ & $2.3(2.1-2.6)$ & \\
\hline Low & $21,641(39.8)$ & $6.5(6.2-6.8)$ & \\
\hline \multicolumn{4}{|c|}{ Municipalities number of residents ${ }^{a}$} \\
\hline $1,000-4,999$ & $4947(9.1)$ & $3.4(2.9-3.9)$ & \multirow[t]{3}{*}{$<0.001$} \\
\hline $5,000-49,999$ & $16,683(30.7)$ & $3.1(2.9-3.4)$ & \\
\hline$\geq 50,000$ & $32,659(60.1)$ & $4.3(4.1-4.5)$ & \\
\hline \multicolumn{4}{|l|}{ District $^{a}$} \\
\hline Jerusalem & 8462 (15.6) & $9.3(8.7-10)$ & \multirow[t]{7}{*}{$<0.001$} \\
\hline South & $3710(6.8)$ & $3.9(3.3-4.5)$ & \\
\hline Ashkelon & $5514(10.1)$ & $3.7(3.2-4.2)$ & \\
\hline Central & $15,169(27.9)$ & $3.6(3.4-4.0)$ & \\
\hline Tel Aviv & $10,457(19.2)$ & $2.2(2.0-2.5)$ & \\
\hline North & 5397 (9.9) & $1.9(1.6-2.3)$ & \\
\hline Haifa & $5582(10.3)$ & $1.1(0.8-1.4)$ & \\
\hline
\end{tabular}

${ }^{a}$ Excluded due to missing data: for municipality ethnicity-3656 individuals, and for municipality religious affiliation-3642 individuals, for socioeconomic status-68 individuals, for number of residents in a municipality—68 individuals, for district—66 individuals
Figure 2 demonstrates the ratio between the percentage of positive serology test results and positive RT-PCR test results at the corresponding time point (at least 4 weeks prior to the serology test date). For each time period, the cumulative seroprevalence was calculated for all participants that underwent the serological test. In addition, the incidence of SARS-CoV-2 in the general population, as was verified by RT-PCR testing, was calculated for each time period. After weighting for age, sex, and district of the survey's population to the respective characteristics of the entire population that underwent RT-PCR testing, a positive correlation was found between the incidence of SARS-CoV-2 and seroprevalence $(\mathrm{r}=0.94, p=0.017)$. The ratio between the seroprevalence and the incidence was 15.7 (3.3 vs. $0.2 \%$, respectively) during the first two weeks of the survey. That ratio decreased over time to a 
Table 2 SARS-CoV-2 seroprevalence by RT-PCR status

\begin{tabular}{llll}
\hline & $\begin{array}{l}\text { Number of partici- } \\
\text { pants n (\%) }\end{array}$ & $\begin{array}{l}\text { Seroprevalence } \\
\%(95 \% \mathrm{CI})\end{array}$ & $P$ value \\
\hline${\text { RT-PCR } \text { status }^{a}}$ & $\mathrm{~N}=54,357$ & & \\
Never performed & $2.1(1.9-2.2)$ & $49,498(91.1)$ & $<0.001$ \\
Performed & $21.9(20.7-23.1)$ & $4859(8.9)$ & \\
RT-PCR result & $\mathrm{N}=4,859$ & & \\
Negative & $6.2(5.4-7.0)$ & $3733(76.8)$ & $<0.001$ \\
Positive & $74(71.3-76.5)$ & $1126(23.2)$ & \\
\hline
\end{tabular}

a3129 participants who underwent RT-PCR test less than 4 weeks prior to serology test were defined as "never performed" RT-PCR testing (of whom, 247 were positive)

Table 3 A multivariate generalized linear mixed model ${ }^{\mathrm{a}}$ for a positive serology test result

\begin{tabular}{lll}
\hline & $\begin{array}{l}\text { Unadjusted odds } \\
\text { ratio }(95 \% \mathrm{CI})\end{array}$ & $\begin{array}{l}\text { Adjusted odds } \\
\text { ratio }{ }^{a} \text { (95\%CI) }\end{array}$ \\
\hline $\begin{array}{l}\text { Time period } \\
\text { 28 June-14 July }\end{array}$ & Ref & Ref \\
14 July-1 August & $1.6(1.4-1.8)$ & $1.7(1.5-2.0)$ \\
1 August-14 August & $3.6(3.1-4.2)$ & $4.5(3.8-5.2)$ \\
14 August-1 September & $4.5(3.9-5.3)$ & $4.6(3.8-5.4)$ \\
1 September-14 September & $6.8(5.6-8.3)$ & $5.4(4.3-6.8)$ \\
RT-PCR & & \\
Never performed & Ref & Ref \\
Performed & $13.3(12.1-14.5)$ & $10.7(9.7-11.8)$ \\
Sex & & \\
Female & Ref & Ref \\
Male & $1.6(1.5-1.7)$ & $1.6(1.4-1.7)$ \\
Age groups (year) & & \\
$0-9$ & $3.4(2.6-4.5)$ & $2.1(1.6-2.8)$ \\
$10-19$ & $4.9(4.0-6.1)$ & $3.3(2.6-4.1)$ \\
$20-29$ & $2.9(2.3-3.6)$ & $2.1(1.7-2.6)$ \\
$30-39$ & $2.2(1.8-2.8)$ & $1.8(1.4-2.3)$ \\
$40-49$ & $2.5(2.0-3.1)$ & $2.2(1.8-2.8)$ \\
$50-59$ & $2.2(1.8-2.8)$ & $2.1(1.7-2.7)$ \\
$60-69$ & $1.5(1.2-1.9)$ & $1.6(1.3-2.0)$ \\
$70+$ & Ref & Ref \\
\hline
\end{tabular}

${ }^{\text {a }}$ The model is fitted with age, sex, time period, and RT-PCR status as fixed effects and municipal strata as a random effect

ratio of 4.6 during the last two weeks of the survey (4.6 vs. $1.0 \%$, respectively)

\section{Discussion}

This nationwide Israeli survey is one of the largest SARSCoV-2 seroprevalence studies conducted thus far throughout the world. Since we predicted a low seroprevalence rate, we favored greater specificity over sensitivity. This goal was achieved by combining two different test kits. Our results indicated a seroprevalence of $3.8 \%$ (weighted $4.6 \%$ ). Since the majority of participants $(89.7 \%)$ underwent the serology test up to August 1, 2020 (the study closure was September 14, 2020), the results mainly reflect the end of the first outbreak until the middle of the second outbreak in Israel. Our findings are important for establishing a baseline level of seroprevalence for future investigations.

While most studies reported no difference in seroprevalence between sexes $[5,6,11,12]$ our finding suggested a 1.6-fold (95\% CI 1.4-1.7) higher prevalence in males compared to females. In agreement with our results, Rosenberg et al. [13] and Iversen et al. [14] also reported higher seroprevalence in males. In contrast, other studies reported a higher seroprevalence in females vs males, $[15,16]$ so the issue remains controversial.

In agreement with other publications, [6, 11, 15] our results indicated differences in prevalence between age groups. We found the highest prevalence rates, both adjusted and unadjusted, in the group aged $10-19$ years (7.8\%). These findings may be explained by the higher numbers of social interactions among adolescence in comparison to other age groups. Children and adolescents are less likely to undergo routine blood tests, therefore, their representation was relatively low in the study (10.8\%) compared to their proportion in the Israeli general population (36\%). However, the survey included 5864 children and adolescents, which is sufficient to draw a representative and reliable conclusion. In agreement with other studies, $[5,7,8,11]$ the lowest seroprevalence was found in participants over the age of 60 years. Since the elderly comprise the major risk group for COVID19 complications, the low prevalence may result from their preference to minimize social interactions. Alternatively, the elderly immune system might be suppressed, whereupon this age group might represent lower or even undetectable levels of antibodies [17].

A municipality's socioeconomic status was highly associated with seropositive results, with a four-fold higher prevalence among low compared to high status. Others have also reported high seroprevalence within populations with low socioeconomic characteristics, such as lower levels of education, [18] lower income, and big household size. [6, 7] Greater exposure to the SARS-CoV-2 virus may be related to higher population density, crowded households, and lack of awareness, all of which result in greater difficulty in observing social distancing. In Israel, ultraorthodox municipalities are characterized by a relatively low socioeconomic status, high population density, and a very unique community structure that makes social distancing difficult to follow, all of which may explain the high seroprevalence detected in ultraorthodox municipalities.

Recent studies have indicated that SARS-CoV-2 IgG antibodies titer continues to rise for three to four weeks after 


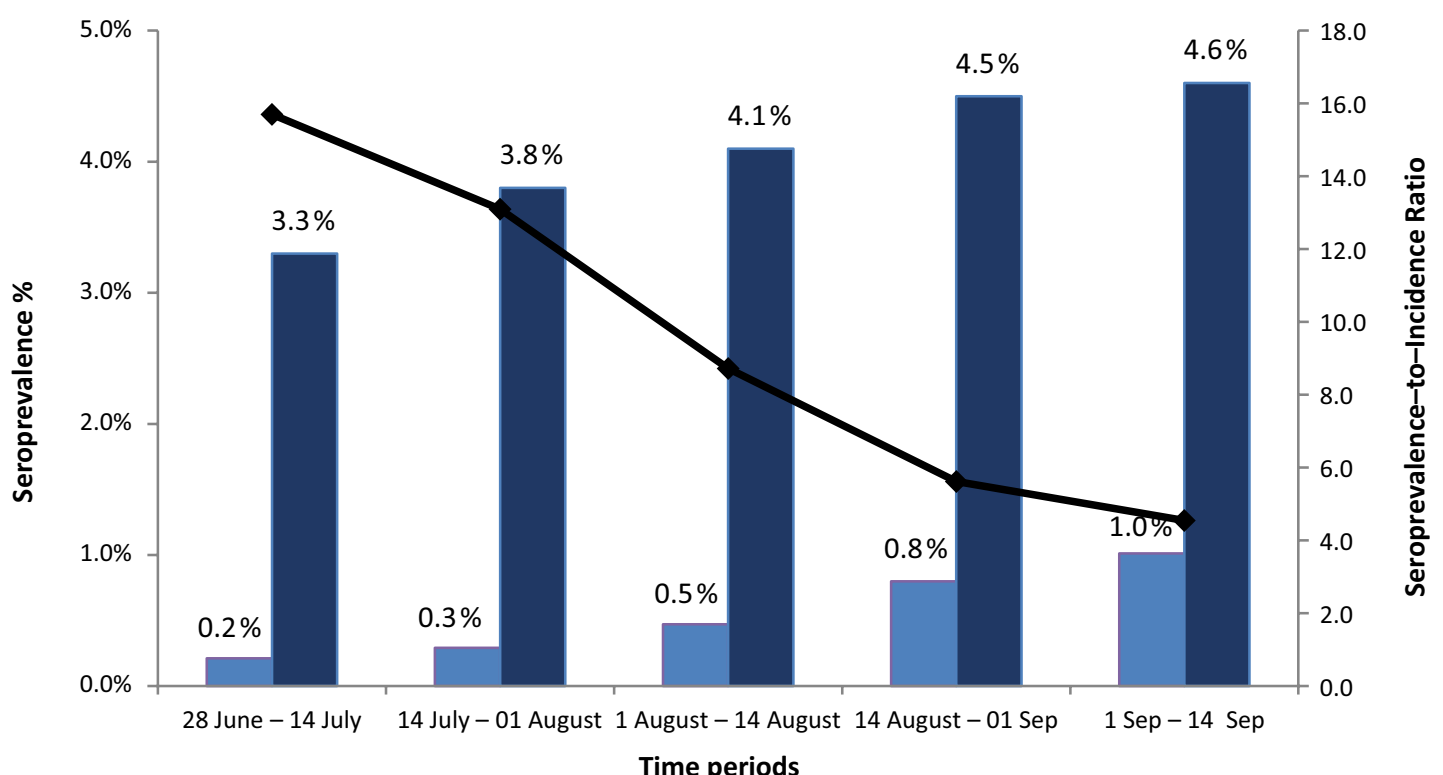

\begin{tabular}{l|c|c|c|c|c|}
\hline Serology date & $28.6-14.7$ & $28.6-1.8$ & $28.6-14.8$ & $28.6-1.9$ & $28.6-14.9$ \\
\hline RT-PCR date & $1.1-14.6$ & $1.1-1.7$ & $1.1-14.7$ & $1.1-1.8$ & $1.1-14.8$ \\
\hline Ratio & $15 \cdot 7$ & $13 \cdot 1$ & 8.72 & 5.62 & 4.55 \\
\multicolumn{4}{r|}{ \% Verified SARS-CoV-2 by RT-PCR Positive IgG by serology }
\end{tabular}

Fig. 2 Seroprevalence ${ }^{\mathrm{a}}$ among survey participants and positive RT-PCR results in the general population by two-week intervals ${ }^{\mathrm{b}}{ }^{\mathrm{a}}$ Adjusted for age, sex, and district. ${ }^{\text {b}}$ For each time period the RT-PCR test date is at least 4 weeks prior to the serology test date

symptom onset [19-23]. In our study, the highest seroprevalence was detected four to eight weeks after a positive RTPCR test result. In accordance, only when a RT-PCR test took place at least four weeks prior to a serology test was it considered as having been performed.

Participants who had undergone RT-PCR testing had an over 10-fold higher likelihood to be seropositive, regardless of their RT-PCR result. During the entire survey, the criterion for undergoing RT-PCR testing in Israel was suspicion for COVID-19, which might explain the higher seropositive rate among the RT-PCR-tested participants. Participants with negative RT-PCR results had a three-fold higher likelihood to be seropositive compared to participants who did not undergo RT-PCR testing. The same trend was observed by others [5, 7]. Ward et al. [7] reported similar results, indicating that individuals with a suspected case of SARS-CoV-2 infection (both by the doctor or by self-report) had a 8.5 -fold higher probability to be seropositive than individuals with no suspicion.

In the current cohort, $6.2 \%$ of the individuals with negative RT-PCR test results were seropositive. This is more likely to be explained by the timing of the negative RT-PCR test rather than receiving false negative RT-PCR test result. Only $74 \%$ of the people with previous positive RT-PCR test results were also seropositive. This may be explained by the reduced combined sensitivity of the test kits (Abbott and DiaSorin), by the reduction in the antibodies titer over time, or by individuals that did not develop antibodies despite of being exposed to SARS-CoV-2 virus [10]. In agreement, several studies reported that asymptomatic carriers are less likely to develop antibodies in comparison to symptomatic patients [23-25]. Another explanation might be the reported rapid decay and short half-life of SARS-CoV-2 antibodies [26, 27].

Israel experienced two COVID-19 outbreaks between the beginning of March 2020 and the end of September 2020. The second wave was prolonged in comparison to the first one and characterized by higher numbers of detected cases. Therefore, both the incidence and seroprevalence rates increased over time.

In the current study, seroprevalence indicated 4.5- to 15.7-fold higher magnitude of infection than that identified by RT-PCR testing. Bendavid et al. [28] reported an estimated 22- to 95-fold higher seroprevalence compared to incidence, and Havers et al. [29] estimated a ratio of 6- to 24-fold seroprevalence over incidence. The relatively low seroprevalence-to-incidence ratio detected in the current study may be due to the high availability and accessibility of 
RT-PCR testing in Israel [3]. At the early stages of the outbreak, the ratio between the two parameters was the highest and it decreased over time. This trend might be explained by the greater availability and accessibility of RT-PCR testing to the Israeli public over time, resulting in a greater number of identified cases. It can be assumed that affording more individuals the possibility of undergoing routine RT-PCR tests would minimize the rate of undetected cases, which would otherwise be revealed only by serology.

Similarly to the sample collection design applied in other seroepidemiologic studyies, $[9,30]$ the population in the current survey is comprised of people undergoing blood tests for any reason. Therefore, despite the large sample size tested, this study might not reliably represent the entire population. However, there is no evidence indicating a higher susceptibility for COVID-19 among individuals undergoing blood tests in HMOs.

Serologic testing is important for evaluating both identified and unidentified infection. It may also be used as an intervention measure, as had been suggested by others [31]. In Israel, serologic test was recognized as a sufficient tool to define a person as having recovered from SARS-CoV-2 infection [32]. Recovery status exempts a seropositive person from 14 days of isolation in cases of close contact with a confirmed SARS-CoV-2 carrier, or after entering the country from abroad. Furthermore, serological diagnosis may support the deployment of employees with a positive serology test to ensure the maintenance of a stable and functional economy. Seroprevalence surveys among healthcare workers and other essential subpopulations are crucial for maintaining a functioning economy in times of COVID-19 outbreaks and may contribute both on personal and national levels.

On December 20, 2020 a wide vaccination program was launched in Israel, aiming at vaccinating the entire population against SARS-CoV-2 within a few months. The number of vaccines already purchased by the Israeli government along with the relatively small population size of the country may enable Israel to be the first country worldwide to achieve herd immunity against COVID-19.

While vaccinations against SARS-CoV-2 are becoming more available worldwide, the use of serology as a tool to manage the pandemic may shift from being a diagnostic tool to a vaccination prioritizing tool. For instance, individuals with a seronegative result may be prioritized over individuals with a seropositive result to achieve a more efficient use of vaccinations and gain "herd immunity" earlier.

In conclusion, our results indicate that the population of Israel is still far from being protected against SARS-Cov-2 by "herd immunity". Additional nationwide surveys are warranted to evaluate the effect of further outbreaks on the seroprevalence in Israel. Consecutive periodical surveys will make it possible to monitor SARS-CoV-2 infection in Israel over time. The findings of this study provide evidence-based data for public health decision-making, not only at the national level but also worldwide. The seroprevalence-toincidence ratio emphasizes the benefits of serology testing, mainly where RT-PCR tests are less available or accessible to the population.

Supplementary Information The online version contains supplementary material available at https://doi.org/10.1007/s10654-021-00749-1.

Author contributions SR was the professional and administrative executive of the project. RR was responsible for data cleaning, statistical analyses and table and figure design. SS was responsible for the conception and design of the study. EK, SR and EA were responsible for the design of the study. SB-S, SH-A, DM and YS coordinated the survey at the HMO level. MF reviewed the statistical analysis. SR and RR wrote the paper. SA, YL, and all other authors contributed to the reviewed of the first draft, and approved the final version and agreed to be accountable for the work.

Funding Not applicable.

Data availability Individual participant data which were collected in the current survey will not be fully available to the public due to legal restrictions and medical confidentiality.

\section{Declarations}

Conflict of interest The authors declared that they have no conflict of interest.

Ethical approval The legal department of the Israeli Ministry of Health approved the study. In Israel, the serology test result has clinical implications for the participant, thus the study was exempt from Helsinki by the Ministry of Health Helsinki committee.

Consent to participate Serology test was offered only to the participants of the current survey. A detailed explanation was given to the participants and they were offered to undergo the blood test at the HMO in which they are insured. By undergoing the blood test, they agreed to participate in the survey.

\section{References}

1. Li Q, Guan X, Wu P, et al. Early transmission dynamics in Wuhan, China, of novel coronavirus-infected pneumonia. N Engl J Med. 2020;382(3):1199-207. https://doi.org/10.1056/NEJMoa2001316.

2. The official data from the Israel Ministry of Health. Open accesses at: https://data.gov.i1/dataset/covid-19. Accessed December 8 2020 .

3. Worldmeter COVID-19 coronavirus pandemic website. https:// www.worldometers.info/coronavirus. Accessed December 62020.

4. The National Insurance Institute of Israel. https://www.btl.gov. il/English\%20Homepage/Insurance/Health\%20Insurance/Pages/ HealthInsuranceLaw.aspx. Accessed December 52020.

5. Pollán M, Pérez-Gómez B, Pastor-Barriuso R, et al. Prevalence of SARS-CoV-2 in Spain (ENE-COVID): a nationwide, populationbased seroepidemiological study. Lancet. 2020;396(10250):53544. https://doi.org/10.1016/S0140-6736(20)31483-5.

6. Hallal PC, Hartwig FP, Horta BL, et al. SARS-CoV-2 antibody prevalence in Brazil: results from two successive 
nationwide serological household surveys. Lancet Glob Health. 2020;8(11):e1390-8. https://doi.org/10.1016/S2214-109X(20) 30387-9.

7. Ward H, Atchison CJ, Whitaker M, et al. Antibody prevalence for SARS-CoV-2 in England following first peak of the pandemic: REACT2 study in 100,000 adults. medRxiv. 2020. https://doi.org/ 10.1101/2020.08.12.20173690.

8. The Public Health Agency of Sweden. First results on antibodies following covid 19 review of blood donors. https://www.folkh alsomyndigheten.se/contentassets/fb47e03453554372ba75ca3d3 a6ba1e7/forekomstren-covid-19-sverige-21-24-april-25-28-maj2020_2.pdf. Accessed December 82020.

9. Bajema KL, Wiegand RE, Cuffe K, et al. Estimated SARS-CoV-2 seroprevalence in the US as of september 2020. JAMA Intern Med. 2020;24:e207976. https://doi.org/10.1001/jamainternmed. 2020.7976

10. Oved K, Liraz O, Shemer-Avni Y, et al. Multi-center nationwide comparison of seven serology assays reveals a SARS-CoV-2 nonresponding seronegative subpopulation. EClinicalMedicine. 2020. https://doi.org/10.1016/j.eclinm.2020.100651.

11. Stringhini S, Wisniak A, Piumatti G, et al. Seroprevalence of antiSARS-CoV-2 IgG antibodies in Geneva, Switzerland (SEROCoVPOP): a population-based study. Lancet. 2020;396(10247):313-9. https://doi.org/10.1016/S0140-6736(20)31304-0.

12. Gallian P, Pastorino B, Morel P, Chiaroni J, Ninove L, de Lamballerie X. Lower prevalence of antibodies neutralizing SARS-CoV-2 in group O French blood donors. Antiviral Res. 2020;181:104880. https://doi.org/10.1016/j.antiviral.2020.104880.

13. Rosenberg ES, Tesoriero JM, Rosenthal EM, et al. Cumulative incidence and diagnosis of SARS-CoV-2 infection in New York. Ann Epidemiol. 2020;48:23-9. https://doi.org/10.1016/j.annep idem.2020.06.004

14. Iversen $\mathrm{K}$, Bundgaard $\mathrm{H}$, Hasselbalch RB, et al. Risk of COVID19 in health-care workers in Denmark: an observational cohort study. Lancet Infect Dis. 2020. https://doi.org/10.1016/S14733099(20)30589-2.

15. Pan Y, Li X, Yang G, et al. Seroprevalence of SARS-CoV-2 immunoglobulin antibodies in Wuhan, China: part of the city-wide massive testing campaign. Clin Microbiol Infect. 2020. https://doi.org/ 10.1016/j.cmi.2020.09.044

16. Vena A, Berruti M, Adessi A, et al. Prevalence of antibodies to SARS-CoV-2 in Italian adults and associated risk factors. J Clin Med. 2020;9(9):2780. https://doi.org/10.3390/jcm9092780.

17. Simon AK, Hollander GA, McMichael A. Evolution of the immune system in humans from infancy to old age. Proc Biol Sci. 2015. https://doi.org/10.1098/rspb.2014.3085.

18. Amorim Filho L, Szwarcwald CL, Mateos SOG, et al. Seroprevalence of anti-SARS-CoV-2 among blood donors in Rio de Janeiro Brazil. Rev Saude Publica. 2020;54:69. https://doi.org/10.11606/ s1518-8787.2020054002643.

19. Korte W, Buljan M, Rösslein M, et al. SARS-CoV-2 IgG and $\mathrm{IgA}$ antibody response is gender dependent; and $\mathrm{IgG}$ antibodies rapidly decline early on. J Infect. 2020. https://doi.org/10.1016/j. jinf.2020.08.032.
20. Sethuraman N, Jeremiah SS, Ryo A. Interpreting diagnostic tests for SARS-CoV-2. JAMA. 2020;323(22):2249-51. https://doi.org/ 10.1001/jama.2020.8259.

21. Rosado J, Pelleau S, Cockram C, et al. Serological signatures of SARS-CoV-2 infection: implications for antibody-based diagnostics. medRxiv. 2020. https://doi.org/10.1101/2020.05.07.20093 963.

22. Solbach W, Schiffner J, Backhaus I, et al. Antibody profiling of COVID-19 patients in an urban low-incidence region in Northern Germany. Front Public Health. 2020. https://doi.org/10.3389/ fpubh.2020.570543.

23. Kowitdamrong E, Puthanakit T, Jantarabenjakul W, et al. Antibody responses to SARS-CoV-2 in patients with differing severities of coronavirus disease 2019. PLoS One. 2020. https://doi.org/ 10.1371/journal.pone.0240502.

24. Yongchen Z, Shen H, Wang X, et al. Different longitudinal patterns of nucleic acid and serology testing results based on disease severity of COVID-19 patients. Emerg Microbes Infect. 2020;9(1):833-6. https://doi.org/10.1080/22221751.2020.17566 99.

25. Long QX, Tang XJ, Shi QL, et al. Clinical and immunological assessment of asymptomatic SARS-CoV-2 infections. Nat Med. 2020;26(8):1200-4. https://doi.org/10.1038/s41591-020-0965-6.

26. Seow J, Graham C, Merrick B, et al. Longitudinal evaluation and decline of antibody responses in SARS-CoV-2 infection. medRxiv. 2020. https://doi.org/10.1101/2020.07.09.20148429.

27. Chen Y, Tong X, Li Y, et al. A comprehensive, longitudinal analysis of humoral responses specific to four recombinant antigens of SARS-CoV-2 in severe and non-severe COVID-19 patients. PLoS Pathog. 2020. https://doi.org/10.1371/journal.ppat.1008796.

28. Bendavid E, Mulaney B, Sood N, et al. COVID-19 antibody seroprevalence in Santa Clara County California. medRxiv. 2020. https://doi.org/10.1101/2020.04.14.20062463.

29. Havers FP, Reed C, Lim T, et al. Seroprevalence of antibodies to SARS-CoV-2 in 10 sites in the United States, March 23-May 12, 2020. JAMA Intern Med. 2020. https://doi.org/10.1001/jamai nternmed.2020.4130.

30. Osborne K, Weinberg J, Miller E. The European sero-epidemiology network. Euro Surveill. 1997;2(4):167. https://doi.org/10. 2807/esm.02.04.00167-en.

31. Weitz JS, Beckett SJ, Coenen AR, et al. Intervention serology and interaction substitution: modeling the role of "shield immunity" in reducing COVID-19 epidemic spread. medRxiv. 2020. https:// doi.org/10.1101/2020.04.01.20049767.

32. The official website of the Israeli Ministry of Health. regulation published by the director of public health services. https://www. gov.il/BlobFolder/legalinfo/bz-306963420-1/he/files_publicatio ns_corona_bz-306963420.pdf. Accessed November 122020.

Publisher's Note Springer Nature remains neutral with regard to jurisdictional claims in published maps and institutional affiliations. 